\title{
Optimal Control of a Formula One Car on a Three-Dimensional Track Part 1: Track Modelling and Identification
}

\author{
Giacomo Perantoni and David J. N. Limebeer
}

\begin{abstract}
The identification of three-dimensional race track models from noisy measured GPS data is treated as a problem in the differential geometry of curves and surfaces. Curvilinear coordinates are adopted to facilitate the use of the track model in the solution of vehicular optimal control problems. Our proposal is to model race tracks using a generalised Frenet-Serret apparatus, so that the track is specified in terms of three displacement-dependent curvatures and two edge variables. The optimal smoothing of the curvature and edge variables is achieved using numerical optimal control techniques. Track closure is enforced through the boundary conditions associated with the optimal control problem. The Barcelona Formula One track is used as an illustrative example.
\end{abstract}

Keywords: road modelling, curvilinear coordinates, differential geometry, Frenet-Serret apparatus, Darboux frame, ribbons, optimal control.

The authors are with the Department of Engineering Science, University of Oxford, Oxford, UK (giacomo.perantoni@gmail.com and david.limebeer@eng.ox.ac.uk.)

\section{Introduction}

$\mathbf{R}$

OAD models are required in a number of applications areas including virtual reality environments, transportation management and planning systems, automated vehicle navigation and geographic information systems. These models are also required in vehicular optimal control problems so that road-related path constraints can be incorporated into the problem. In the context of road racing these optimal control problems usually relate to minimum-lap-time optimal control problems. Road-related path constraints are enforced by ensuring that the car's mass centre, or its road wheels, remain within the track boundaries. In [1] a 2-dimensional (flat) road model is considered and any deviations from the road are penalised by a performance index term involving the distance between the car's mass centre and the centre of the road. These ideas are developed in [2], where a flat road is again used with state constraints applied to a finite number of check points that are distributed along the periphery of the track. In this work it is assumed that if these boundary constraints are properly located, they can be used to represent the track boundaries everywhere. Much the same ideas are used in [3], where a flat track is again assumed. Building on the work in [4], the study presented in [5] makes use of a 2-dimensional curvilinear-coordinate-based track description.

As was observed in [2], measurement noise makes it difficult to deduce track parameters by numerically integrating measured telemetry data. Key difficulties relate to errors in the kinematic models used and drift in the integration processes. In combination, these errors can result in a displacement mismatch between the track 
start and end points of several tens (and even hundreds) of meters. In order to overcome this problem an ad-hoc error-compensation procedure was used to enforce track closure [2]. An intrinsic coordinate description of the road is adopted in [6], where the absolute orientation of the road centreline tangent is used instead of its curvature. The road model is employed for minimum manoeuvre time calculations using convex optimization in a linear model-predictive-control framework. The disadvantages associated with absolute coordinate systems have been recognised by several researchers [4-6]. In the minimum-time-manoeuvring literature tracks are usually modelled as sequences of straight sections, clothoids and circular sections, which can guarantee both the continuity of the curvature and the closure of a circuit. A downside of this approach is that the geometry of real tracks cannot be reproduced exactly, and the modelling process requires the manual segmentation of the track into sections of different type on the basis of the road curvature. In the two-dimensional case the circuit closure problem has been solved using a curvilinear-coordinate-based model with the track curvature deduced from the solution of an optimal control problem that includes a boundary closure constraint [5].

Ribbons or strips are central to road models and provide a differential-geometric description of navigable road surfaces. As is explained in [7], general surfaces can be studied in terms of strips or ribbons — think of the ribbon of peel one discards after removing it from the surface of an apple. In the same way that surfaces can be studied in terms of ribbons, ribbons can be studied in terms of curves; one such curve could be the strip's centre line. These curves are also referred to as the spine of the ribbon; the spine need not be the centre line. The general idea is to describe the surface, or ribbon, in terms of tangent planes computed along the spine. Some of these modelling ideas are illustrated in Figure 1, which will later be returned to when making these ideas precise. Ribbon-based models

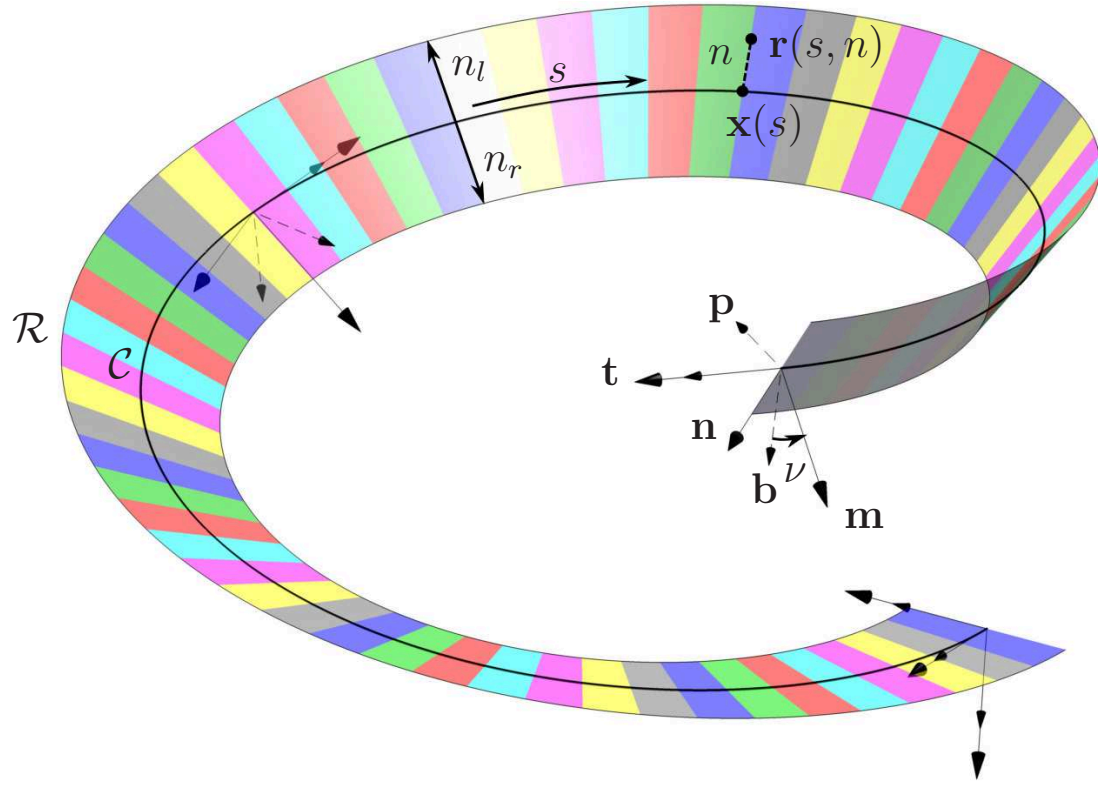

Fig. 1. A ribbon $\mathcal{R}$ generated by a spine curve $\mathcal{C}$. The spine is described in terms of its torsion and curvature, while the camber of the ribbon comes from the twist angle $\mathrm{V}(s)$.

have been used to describe DNA, polymers and other chain-like molecules and some of their properties [8-11]. For our purposes these papers play a useful role by describing some of the differential-geometric ideas that we will use here; the account given in [11] is particularly useful.

There are various classes of ribbon-based road models distinguished by their purpose and the type of measured data used. A first family of techniques address the problem of estimating the road geometry ahead of a moving vehicle, which are designed for use in real-time driving assistance systems. These systems exploit road boundary information that is gathered by real-time look-ahead sensors. Real-time applications limit the compu- 
tational complexity of the estimation techniques used, with some form of recursive estimation procedure usually preferred. In [12], the concurrent estimation of the parameters in a clothoid-based road model, and the relative position of a vehicle with respect to the road are considered. Kalman filtering techniques are used in the recursive estimation of the clothoid parameters and the vehicle state. This paper also reports on experimental results that show good accuracy in the estimation of the road curvature for a series of corners in the Neubiberg test track. Other papers based on structured road models and Kalman filtering followed this work, including road models based on clothoids [13], sequences of clothoids [14,15], polynomials and piecewise polynomials [16]. Following an overview of the techniques used for real-time road estimation, [16] proposes a road model based on the use of three-dimensional B-spline representations of the road boundaries. This appears to be the only study on real-time road estimation that takes account for both cambered surfaces and variable road width, and arguably represents the closest point of contact with the research presented here. More sophisticated approaches propose the concurrent estimation of road parameters for multi-road models $[17,18]$, and provide strategies for the dynamic selection of the most suitable road geometry. The off-line method proposed in [19] assumes knowledge of a complete set of measured road data. The road parameters are estimated through least-square error minimisation that considers a look-ahead window of 5-6 seconds, with the distance based on the vehicle speed. The underlying road model is planar and is based on an approximation of a clothoid; road width variations are not considered.

A second class of road estimation methods focusses on artificial vision and pattern recognition techniques for the construction of road networks, with applications in driving simulation in virtual environments, and traffic simulation. These methods were comprehensively surveyed in [20] and more recently reviewed in [21]. In [21] the road is modelled as a ribbon using curvilinear-coordinates. A Kalman filter, based on a kinematic road model, is used to predict future road centre-line positions from observations and the current road state. It was observed that most public roads tend to be 'smooth' and that the curvature of their centre line can usually be bounded in proportion to the road width. This has the useful side effect of excluding possible singularities from ribbon-based road models.

Central to the methods used in the second class of road estimation methods are image-processing techniques for road extraction. In this context work road geometry accuracy is not deemed important, since it is not used in detailed vehicle dynamics studies. However, the need for three-dimensional road models is recognised in the context of road network modelling. A ribbon road representation that relies on an annotated three-dimensional curve, which acts as the spine, is proposed [22].

In the present paper we introduce formal differential-geometric concepts into road modelling integrating and extending ideas spread across different application areas in order to achieve the following objectives:

1. model an arbitrary, closed, three-dimensional road circuit, allowing for curvature, camber and elevation changes, as well as a variable track width;

2. propose an optimal estimation procedure that provides a smooth parameterisation of the road model from noisy data relating to the absolute positions of the track boundaries;

3. remove assumptions on the expected geometry of the road such as, for example, assuming that it is made up of sequences of straights, clothoids and circles. This reduces the errors and complexity associated with road segmentation (into a preassigned set of curve types);

4. enforce track closure as a boundary condition in an optimal estimation problem.

In common with the work in [5], this paper does not consider short-wavelength road features. We will assume that the road surface is locally 'flat', and that it can be modelled using boundary information only.

The differential geometry of curves and ribbons in summarised in Section 2. We begin with a summary of some of the standard properties of cross products and skew-symmetric matrices in Section 2.1. The geometry of curves is summarised in Section 2.2 with the differential-geometric properties of ribbons given in Section 2.3. The relationship between these differential-geometric curvature ideas and Euler angles is summarised in Section 2.4. The optimal estimation problem is described in Section 3. This includes a finite-difference estimate of the track boundaries which appears in Section 3.1, a summary of the influences of measurement noise is given in Section 3.2. 
The optimal estimation problem is formulated as an optimal control problem in Section 3.3. The results are given in Section 4. A ribbon based on the Lemniscate of Bernoulli is studied in Section 4.1 and the Circuit de Catalunya is modelled in Section 4.2. The conclusions appear in Section 5.

\section{Geometry of Ribbons}

The purpose of this section is to assemble a number of geometric ideas that will be used to describe threedimensional roads. All of these concepts can be found in the classical differential geometry literature; see for example [23] and [24]. In order to minimise notational clutter, we will usually suppress independent variables. These variables will only be given explicitly when defining new quantities, or when confusion or ambiguity could result from their omission. We use boldface characters to represent three-dimensional vectors and upper case characters for matrices. Calligraphic characters represent sets and bold uppercase characters are used for points.

\subsection{Rotations and Cross Products}

A position vector $\mathbf{r}_{B}$ that is expressed in the coordinate system of a rotating body $B$ can be expressed in an inertial frame using the transformation

$$
\mathbf{r}_{I}=R \mathbf{r}_{B}
$$

where $R$ is a rotation matrix that maps the body-fixed axis system into the inertial coordinate system.

All rotation matrices satisfy $R R^{T}=I$ with the added constraint that $\operatorname{det}(R)=1$; rotation matrices preserve orientations and are therefore a subset of the orthogonal matrices. Every rotation matrix can be expressed in terms of an axis-of-rotation unit-vector $\mathbf{n}$ and a rotation angle $\varphi$. For any rotation matrix $R$ there exists a pair $\mathbf{n}$ and $\varphi$ such that $R=R(\mathbf{n}, \varphi)$, in which

$$
R(\mathbf{n}, \varphi)=\cos (\varphi) I+(1-\cos (\varphi)) \mathbf{n n}^{T}+\sin (\varphi) S(\mathbf{n})
$$

The skew-symmetric matrix $S(\mathbf{n})$ is defined by

$$
S(\mathbf{n})=\left[\begin{array}{ccc}
0 & -n_{z} & n_{y} \\
n_{z} & 0 & -n_{x} \\
-n_{y} & n_{x} & 0
\end{array}\right],
$$

where $n_{x}, n_{y}$ and $n_{z}$ are the $x-, y$ - and $z$-axis components of $\mathbf{n}$. Equation (2) is often referred to as the Rodrigues' rotation formula; $R(\mathbf{n}, \varphi)$ performs a rotation about an arbitrary unit vector $\mathbf{n}$ through angle $\varphi$. Since $\mathbf{n}$ is of unit length, $R(\mathbf{n}, \varphi)$ is determined by $\varphi$ and any two components of $\mathbf{n}$ (three parameters).

If in some frame of reference the vectors $\mathbf{a}$ and $\mathbf{b}$ have component-wise representations $\left[a_{1} a_{2} a_{3}\right]^{T}$ and $\left[b_{1} b_{2} b_{3}\right]^{T}$, then their cross product is given by

$$
\mathbf{a} \times \mathbf{b}=\left[\begin{array}{l}
a_{2} b_{3}-a_{3} b_{2} \\
a_{3} b_{1}-a_{1} b_{3} \\
a_{1} b_{2}-a_{2} b_{1}
\end{array}\right] .
$$

The $i^{\text {th }}$ component of the cross product can also be expressed in terms of the Levi-Civita symbol $\varepsilon_{i j k}$ as

$$
(\mathbf{a} \times \mathbf{b})_{i}=\varepsilon_{i j k} a_{j} b_{k} ;
$$


the summation symbols (associated with $j$ and $k$ ) are omitted in the Einstein summing convention.

Since the cross product (by a) is a linear operation, it has a matrix representation $S(\mathbf{a})$, where $S(\cdot)$ is defined in (3); $\mathbf{a} \times \mathbf{b}=S(\mathbf{a}) \mathbf{b}$. It is important to note that rotations commute with the cross product; that is $R(\mathbf{a} \times \mathbf{b})=$ $(R \mathbf{a}) \times(R \mathbf{b})^{1}$. A standard calculation shows that

$$
R S(\mathbf{a}) R^{T}=S(R \mathbf{a})
$$

We will use the properties of cross products to derive a number of formulae relating to motions in rotating reference frames. For any rotation matrix $R(s)$, there holds $d\left(R R^{T}\right) / d s=\dot{R} R^{T}+\left(\dot{R} R^{T}\right)^{T}=0^{2}$, which implies $\dot{R} R^{T}=-\left(\dot{R} R^{T}\right)^{T}$, whence $\dot{R} R^{T}$ is skew symmetric. Since $R^{T} \dot{R}=R^{T}\left(\dot{R} R^{T}\right) R, R^{T} \dot{R}$ is skew-symmetric too.

In the case that $\mathbf{r}_{B}$ is constant, it follows from (1) that $\dot{\mathbf{r}}_{I}=\dot{R} \mathbf{r}_{B}=\dot{R} R^{T} \mathbf{r}_{I}=S\left(\Omega_{I}\right) \mathbf{r}_{I}=\Omega_{I} \times \mathbf{r}_{I}$, where $\Omega_{I}$ is the angular velocity of the moving frame (expressed in inertial coordinates). Alternatively, $\dot{\mathbf{r}}_{I}=R\left(R^{T} \dot{R}\right) R^{T} \mathbf{r}_{I}=$ $S\left(R \Omega_{B}\right) \mathbf{r}_{I}$, where $R^{T} \dot{R}=S\left(\Omega_{B}\right)$ with $\Omega_{B}$ is the angular velocity of the moving frame expressed in its own coordinate system. Finally, suppose that the columns of $R$ are the basis vectors of a rotating trihedral coordinate system (expressed in an inertial frame). Then the way that this trihedron moves relative to the inertial frame is described by $\dot{R}=\left(\dot{R} R^{T}\right) R=R\left(R^{T} \dot{R}\right)$, and so $\dot{R}=S\left(\Omega_{I}\right) R$, or alternatively $\dot{R}=R S\left(\Omega_{B}\right)$.

\subsection{Curves}

Consider the parametric representation of the arc of a curve

$$
\mathcal{C}=\left\{\mathbf{x}(s)=[x(s) y(s) z(s)]^{T} \in \mathbb{R}^{3}: s \in\left[s_{0}, s_{f}\right]\right\}
$$

in which the parameter $s$ is the arc length of $\mathcal{C}$. We assume that each element of $d^{3} \mathbf{x} / d s^{3}$ is continuous and that $d^{2} \mathbf{x} / d s^{2}$ is not identically 0 for any $s$. The curve is deemed simple, if any point on the curve corresponds to a single value of $s$.

A moving trihedron associated with $\mathcal{C}$ is defined by a triple of unit vectors $\mathbf{t}(s), \mathbf{p}(s)$ and $\mathbf{b}(s)$ that form a right-handed coordinate system with origin $\mathbf{x}$. The unit vector $\mathbf{t}$ is tangent to $C$ and is defined by $\mathbf{t}=\dot{\mathbf{x}}$. The unit vector $\mathbf{p}$ is the principal normal to $C$ and is defined as $\mathbf{p}=\dot{\mathbf{t}} /|\dot{\mathbf{t}}|$. The unit vector $\mathbf{b}$ is called the binormal to the curve, and is defined by the vector product $\mathbf{t} \times \mathbf{p}$. A pictorial representation of $\mathcal{C}$ and its moving trihedron is shown in Figure 1.

The curvature vector of $\mathcal{C}$ is defined as the rate of change of the tangent vector, $\kappa(s)=\dot{\mathbf{t}}$. We define the curvature as the scalar product $\kappa(s)=\kappa \cdot \mathbf{p}$, which is a measure of the deviation of $\mathcal{C}$ from a straight line; it follows that $\kappa=\kappa \mathbf{p}$, where

$$
\kappa=|\ddot{\mathbf{x}}|
$$

The radius of curvature is defined as $\rho(s)=1 / \kappa$. The centre of curvature is given by $\mathbf{C}(s)=\mathbf{x}+\rho \mathbf{p}$. The torsion of $\mathcal{C}$ measures the direction and extent to which the curve deviates from its osculating plane tp. The torsion is defined as $\tau(s)=-\mathbf{p} \cdot \dot{\mathbf{b}}$; the curvature and torsion are also known as the first curvature and second curvature respectively. Finally:

$$
\tau=\dot{\mathbf{x}} \cdot(\ddot{\mathbf{x}} \times \dddot{\mathbf{x}})|\ddot{\mathbf{x}}|^{-2}
$$

\footnotetext{
${ }^{1}$ If $\mathbf{a} \times \mathbf{b}=\mathbf{c}$, then $R(\mathbf{a} \times \mathbf{b})=R \mathbf{c}$. If $\mathbf{a}^{\prime}=R \mathbf{a}, \mathbf{b}^{\prime}=R \mathbf{b}$ and $\mathbf{c}^{\prime}=R \mathbf{c}$, then $\mathbf{a}^{\prime} \times \mathbf{b}^{\prime}=\mathbf{c}^{\prime}$, since the relative orientations and lengths of these vectors have not changed. Consequently $(R \mathbf{a}) \times(R \mathbf{b})=R \mathbf{c}=R(\mathbf{a} \times \mathbf{b})$.

${ }^{2}$ Throughout this paper we will assume that we are dealing with constant-speed curves and so derivatives with respect to time $t$ and arc length $s$ are equivalent; $\frac{d s}{d t}=1$. We will use a dot to denote derivatives with respect to either variable.
} 
which is why $C$ is assumed 2-smooth [23].

The orientation of the moving trihedron is defined by the three-dimensional rotation matrix

$$
R(s)=[\mathbf{t} \mathbf{p} \mathbf{b}]
$$

The curvature and torsion define the angular velocity the moving trihedron through the kinematic equations known as the Frenet-Serret formulae:

$$
\dot{R}=R\left[\begin{array}{ccc}
0 & -\kappa & 0 \\
\kappa & 0 & -\tau \\
0 & \tau & 0
\end{array}\right]=R S\left(\Omega_{B}\right)
$$

It is immediate from (3) that each column of (9) has a kinematic interpretation of the form $\mathbf{v}=\Omega \times \mathbf{r} ; \mathbf{v}$ is a velocity vector, $\mathbf{r}$ is a position vector and $\Omega$ is an angular velocity vector of the type familiar in classical mechanics. More particularly, $\dot{\mathbf{t}}=\kappa \mathbf{p}, \dot{\mathbf{p}}=\tau \mathbf{b}-\kappa \mathbf{t}$ and $\dot{\mathbf{b}}=-\tau \mathbf{p}$. The angular velocity vector of the moving trihedron is known as the Darboux vector and is given by $\Omega_{B}=\tau \mathbf{t}+\kappa \mathbf{b}$.

By imposing initial conditions on the position $\mathbf{x}\left(s_{0}\right)$ and the orientation $R\left(s_{0}\right)$ of the body-fixed reference frame, the curve $C$ is generated by

$$
\left.\begin{array}{l}
R=\int_{s_{0}}^{s} \dot{R} d s \\
\mathbf{x}=\int_{s_{0}}^{s} \mathbf{t} d s
\end{array}\right\}
$$

in which $\mathbf{t}$ is the first column of $R$ in (8). The Frenet-Serret equations can be used as a parameterisation of a curve in terms of its curvature and torsion.

\subsection{Ribbons}

A ribbon $\mathcal{R}$ can be created by augmenting $\mathcal{C}$ with notions of 'width' and 'twist'. Both of these quantities will be defined in terms of a unit camber vector $\mathbf{n}(s)$, which lies in the plane of the ribbon and is orthogonal to the tangent vector $\mathbf{t}$. We will represent the ribbon parametrically as:

$$
\mathcal{R}=\left\{\mathbf{r}(s, n)=\mathbf{x}(s)+\mathbf{n}(s) n \in \mathbb{R}^{3}: s \in\left[s_{0}, s_{f}\right], n \in\left[n_{l}(s), n_{r}(s)\right]\right\},
$$

in which $s$ is the arc length and $n$ is a lateral offset in the ribbon plane. Lateral displacements on the ribbon surface are measured from the spine in the direction of $\mathbf{n}$. The width of the ribbon at each $s$ is given by $\left|n_{r}(s)-n_{l}(s)\right|$. This parameterisation will accommodate variable width ribbons that need not be symmetric around the spine curve $\mathcal{C}$. If $\mathbf{r}(s, n)$ is a point in $\mathcal{R}$, then we will assume that there is a one-to-one mapping between this point and the parameter domain. The curves on $\mathcal{R}$ corresponding to constant $s$, or constant $n$, are called coordinate curves; the coordinate curve corresponding to $n=0$ is the spine of the ribbon. The spine is modelled as a simple curve $C$ and can be described in terms of the geometric entities introduced in Section 2.2. The camber vector lies in the plane normal to the spine's tangent vector $\mathbf{t}$ and can therefore be described in terms of $\mathbf{p}$, $\mathbf{b}$ and a twist angle $v(s)$ as follows

$$
\mathbf{n}=\mathbf{p} \cos v-\mathbf{b} \sin v .
$$


The twist rate is $\dot{\mathrm{v}}$. A unit vector $\mathbf{m}(s)$ that is normal to the ribbon surface is defined to complete a right-handed axis system with $\mathbf{t}$ and $\mathbf{n}$, whence $\mathbf{m}=\mathbf{t} \times \mathbf{n}$. See Figure 1 for an illustration of a ribbon and these quantities.

The Frenet-Serret formulae can be used to describe the spine, but they do not have the flexibility to describe the ribbon itself. Developing again a kinematic interpretation of the problem, a moving triad $\mathbf{t} \mathbf{n} \mathbf{m}$, which we call the ribbon trihedron, is used to describe the evolution of the spine and the ribbon itself. The ribbon trihedron is sometimes called the Darboux frame of $\mathcal{C}$ on $\mathcal{R}$. The angular velocity of the reference frame fixed to the ribbon trihedron is given by the sum of the Darboux vector and the twist rate:

$$
\Omega(s)=[\tau+\dot{v}] \mathbf{t}+\kappa \mathbf{b} .
$$

The ribbon angular velocity vector $\Omega(s)$ can be expressed in the ribbon trihedron through a coordinate transformation from the curve trihedron [9], [10] and [11]:

$$
\Omega_{B}=\left[\begin{array}{ccc}
1 & 0 & 0 \\
0 & \cos v & \sin v \\
0 & -\sin v & \cos v
\end{array}\right]\left[\begin{array}{c}
\tau+\dot{v} \\
0 \\
\kappa
\end{array}\right]=\left[\begin{array}{c}
\tau+\dot{v} \\
\kappa \sin v \\
\kappa \cos v
\end{array}\right]
$$

where $\Omega_{B}$ is expressed in the ribbon trihedron.

The angular velocity vector $\Omega_{B}$ is thus a vector in $\mathbb{R}^{3}$, which has a skew-symmetric matrix representation $S\left(\Omega_{B}\right)$ :

$$
\Omega_{B}=\left[\begin{array}{c}
\Omega_{x} \\
\Omega_{y} \\
\Omega_{z}
\end{array}\right], S\left(\Omega_{B}\right)=\left[\begin{array}{ccc}
0 & -\Omega_{z} & \Omega_{y} \\
\Omega_{z} & 0 & -\Omega_{x} \\
-\Omega_{y} & \Omega_{x} & 0
\end{array}\right]
$$

where $\Omega_{x}, \Omega_{y}$ and $\Omega_{z}$ are known as the relative torsion, the normal curvature and the geodesic curvature, respectively. The matrix $S\left(\Omega_{B}\right)$, also known as the Cartan matrix [7], is a generalisation of the Frenet-Serret formulae to the case of ribbons and

$$
\dot{R}=R S\left(\Omega_{B}\right)
$$

As before, the body-fixed reference frame translates at unit speed in the tangent axis direction, starting from the initial conditions $\mathbf{x}\left(s_{0}\right)$ and $R\left(s_{0}\right)$. The ribbon $\mathcal{R}$ is thus given in integral form by

$$
\left.\begin{array}{l}
R=\int_{s_{0}}^{s} \dot{R} d s \\
\mathbf{r}=\int_{s_{0}}^{s} \mathbf{t} d s+n \mathbf{n},
\end{array}\right\}
$$

in which $\dot{R}$ is given by (16). The generalised Frenet-Serret equations are used as a differential-geometric description of ribbons in terms of their generalised curvature vector $\Omega_{B}$ and their width parameters.

\subsection{Euler angles}

The ribbon-based model of the road is given in (17), with the evolution of the ribbon trihedron, as a function of arc length, coming from integrating a $3 \times 3$ rotation matrix. 
Since $R R^{T}=I$ for any rotation matrix $R$, there are six constraints on its nine entries so that all rotation matrices can be described in terms of three parameters. One such parameterisation is based on Euler angles, wherein a general rotation matrix is decomposed into a sequence of three elementary rotations about body-fixed axes. Each elementary rotation is described by a single Euler angle. Discounting degenerate cases corresponding to consecutive rotations about the same axis, twelve possibilities remain such as $x y x, x y z, y x y$ and so on. As is well known, the three elementary rotations are defined by (2):

$$
\begin{aligned}
& R_{x}(\phi)=R\left(\mathbf{e}_{x}, \phi\right)=\left[\begin{array}{ccc}
1 & 0 & 0 \\
0 & \cos \phi & -\sin \phi \\
0 & \sin \phi & \cos \phi
\end{array}\right], \\
& R_{y}(\mu)=R\left(\mathbf{e}_{y}, \mu\right)=\left[\begin{array}{ccc}
\cos \mu & 0 \sin \mu \\
0 & 1 & 0 \\
-\sin \mu & 0 & \cos \mu
\end{array}\right], \\
& R_{z}(\theta)=R\left(\mathbf{e}_{z}, \theta\right)=\left[\begin{array}{ccc}
\cos \theta-\sin \theta & 0 \\
\sin \theta & \cos \theta & 0 \\
0 & 0 & 1
\end{array}\right],
\end{aligned}
$$

in which the subscripts represent the axes around which rotations occur and the arguments the associated angles. The sequence of rotations must be chosen in a way best suited to the problem. One of the key issues relates to the fact that every sequence of rotations has an associated subspace of singular configurations in which the values of the Euler angles are not uniquely defined for the given rotation. This happens when the second rotation aligns the first and the third rotation axes, and the first and third Euler angles can be chosen arbitrarily. Singular configurations may be an issue when the problem of finding the Euler angles corresponding to a given rotation matrix is considered. These are known as inverse kinematics problems and arise in robotics.

In our road surface modelling work we will use a zyx Euler angle sequence in which the first rotation $\theta$ (yaw) is associated with the direction of the road in the $x y$ inertial plane, the second angle $\mu$ (pitch) describes the road inclination in the direction of travel, while the third angle $\phi$ (roll) describes the road camber. Singular configurations for this rotation sequence correspond to a road with a pitch angle of $\pm \pi / 2$, which does not exist in usable driving environments. For this choice of Euler angles the rotation matrix is parameterised as

$$
R=R_{z}(\theta) R_{y}(\mu) R_{x}(\phi)=\left[\begin{array}{ccc}
\mathrm{c}_{\theta} \mathrm{c}_{\mu} \mathrm{c}_{\theta} \mathrm{s}_{\mu} \mathrm{s}_{\phi}-\mathrm{s}_{\theta} \mathrm{c}_{\phi} \mathrm{c}_{\theta} \mathrm{s}_{\mu} \mathrm{c}_{\phi}+\mathrm{s}_{\theta} \mathrm{s}_{\phi} \\
\mathrm{s}_{\theta} \mathrm{c}_{\mu} \mathrm{s}_{\theta} \mathrm{s}_{\mu} \mathrm{s}_{\phi}+\mathrm{c}_{\theta} \mathrm{c}_{\phi} \mathrm{s}_{\theta} \mathrm{s}_{\mu} \mathrm{c}_{\phi}-\mathrm{c}_{\theta} \mathrm{s}_{\phi} \\
-\mathrm{s}_{\mu} & \mathrm{c}_{\mu} \mathrm{s}_{\phi} & \mathrm{c}_{\mu} \mathrm{c}_{\phi}
\end{array}\right]
$$

in which 's' and 'c' correspond to the sine and cosine of the subscript angles.

Suppose $\mathbf{P}$ is a point stationary in a moving reference frame. The coordinates of the position vector of this point, when expressed in the moving frame, are constant and denoted $\mathbf{r}_{B}$. In an inertial reference frame this point is described by the position vector $\mathbf{r}_{I}=R \mathbf{r}_{B}$. The velocity of this point (expressed in the inertial frame) is found as follows:

$$
\dot{\mathbf{r}}_{I}=\dot{R} \mathbf{r}_{B}=\dot{R} R^{T} \mathbf{r}_{I}=S\left(\Omega_{I}\right) \mathbf{r}_{I}=\Omega_{I} \times \mathbf{r}_{I}
$$

The angular velocity vector $\Omega_{I}$ is expressed in the inertial frame; $\Omega_{B}=R^{T} \Omega_{I}$ for the moving frame. If we apply these ideas to the rotation matrix given in (20), an equivalent expression for the speed of $\mathbf{P}$ can be derived as 
follows:

$$
\begin{aligned}
\dot{\mathbf{r}}_{I} & =\dot{R} R^{T} \mathbf{r}_{I} \\
& =\left(\dot{R}_{z} R_{y} R_{x}+R_{z} \dot{R}_{y} R_{x}+R_{z} R_{y} \dot{R}_{x}\right) R_{x}^{T} R_{y}^{T} R_{z}^{T} \mathbf{r}_{I} \\
& =\left(\dot{R}_{z} R_{z}^{T}+R_{z} \dot{R}_{y} R_{y}^{T} R_{z}^{T}+R_{z} R_{y} \dot{R}_{x} R_{x}^{T} R_{y}^{T} R_{z}^{T}\right) \mathbf{r}_{I} \\
& =\left(S\left(\dot{\theta} \mathbf{e}_{z}\right)+R_{z} S\left(\dot{\mu} \mathbf{e}_{y}\right) R_{z}^{T}+R_{z} R_{y} S\left(\dot{\phi} \mathbf{e}_{x}\right) R_{y}^{T} R_{z}^{T}\right) \mathbf{r}_{I} \\
& =\left(\dot{\theta} \mathbf{e}_{z}+\dot{\mu} R_{z} \mathbf{e}_{y}+\dot{\phi} R_{z} R_{y} \mathbf{e}_{x}\right) \times \mathbf{r}_{I},
\end{aligned}
$$

where $\mathbf{e}_{x}, \mathbf{e}_{y}$ and $\mathbf{e}_{z}$ are unit vectors associated with rotations around the $x$-, $y$ - and $z$-axes. Since the equalities (20) and (21) must hold for all points $\mathbf{P}$, there obtains

$$
\Omega_{B}=R^{T}\left(\dot{\theta} \mathbf{e}_{z}+\dot{\mu} R_{z} \mathbf{e}_{y}+\dot{\phi} R_{z} R_{y} \mathbf{e}_{x}\right)=J[\dot{\phi} \dot{\mu} \dot{\theta}]^{T}
$$

with

$$
J=\left[\mathbf{e}_{x} R_{x}^{T} \mathbf{e}_{y} R_{x}^{T} R_{y}^{T} \mathbf{e}_{z}\right]
$$

As in all mechanics problems, the idea is to replace the $3 \times 3$ matrix in (16) with three scalar differential equations in the Euler angles. In this case the kinematic parameterisation of the ribbon (17) becomes

$$
\left.\begin{array}{rl}
\theta(s) & =\int_{s_{0}}^{s} \dot{\theta}(s) d s, \\
\mu(s) & =\int_{s_{0}}^{s} \dot{\mu}(s) d s, \\
\phi(s) & =\int_{s_{0}}^{s} \dot{\phi}(s) d s, \\
\mathbf{r}(s, n) & =\int_{s_{0}}^{s} \mathbf{t}(s) d s+n \mathbf{n}(s),
\end{array}\right\}
$$

where $\mathbf{t}$ and $\mathbf{n}$ are the first and second columns, respectively, of the rotation matrix $R$ as given in (19), and the initial conditions $\theta\left(s_{0}\right), \mu\left(s_{0}\right)$ and $\phi\left(s_{0}\right)$ are found by solving the inverse problem for $R\left(s_{0}\right)$.

The generalised curvature vector components are determined using (22), in which the Jacobian matrix $J$ for the selected Euler-angle convention is

$$
J(s)=\left[\begin{array}{rrr}
1 & 0 & -s_{\mu} \\
0 & c_{\phi} & c_{\mu} s_{\phi} \\
0 & -s_{\phi} & c_{\mu} c_{\phi}
\end{array}\right],
$$

as derived from (23).

\section{Optimal Control}

The overarching aim of the work presented in this paper is to use measured data to find a continuous curvilinearcoordinate-based road model that can be used in the solution of vehicular optimal control problems. We will assume that the road-related measurement data comprises discrete GPS coordinate measurements of the track 
boundaries. These measurements will typically be noisy and need not be equispaced. Let us suppose that the lefthand boundary measurements are given by $\mathcal{B}_{l}=\left\{\mathbf{b}_{l i} \in \mathbb{R}^{3}: i=1 \ldots N\right\}$. The right-hand boundary measurements are given by $\mathcal{B}_{r}=\left\{\mathbf{b}_{r i} \in \mathbb{R}^{3}: i=1 \ldots N\right\}$; we assume equal numbers of right- and left-hand boundary points ${ }^{3}$.

The track centre line can be found by a simple nearest-neighbour search and averaging calculation. Suppose the first right-hand boundary point $\mathbf{b}_{r 1}$ is selected. One then searches the left-hand boundary measurement set for its closest neighbour, which we call the first left-hand boundary point $\mathbf{b}_{l 1}$. We then say $\mathbf{c}_{1}=\left(\mathbf{b}_{l 1}+\mathbf{b}_{r 1}\right) / 2$ is the first track centre point. By repeating this process for all the right-hand boundary points, estimates for the track centre line $\mathcal{C}=\left\{\mathbf{c}_{i} \in \mathbb{R}^{3}: 1 \ldots N\right\}$ can be found. The arc lengths associated with each point are found by summing the incremental distances between successive measurement points. Suppose that the GPS coordinates of $\mathbf{c}_{i}$ are known and that $\Delta_{i}:=\left|\mathbf{c}_{i+1}-\mathbf{c}_{i}\right|$ for $i=1, \ldots N-1$, then the arc lengths associated with each point are given by $s_{j}=\sum_{i=1}^{j-1} \Delta_{i}$ for $j=1, \ldots N-1$. If the track is closed we set $\Delta_{N}:=\left|\mathbf{c}_{1}-\mathbf{c}_{N}\right|$, with $s_{N}$ the track length in this case.

\subsection{Finite-differencing}

In the absence of noise, the track might be described in terms of curvature variables determined by numerical differentiation. If Euler forward-differencing is used, an approximate ribbon parameterisation can be found using

$$
\begin{aligned}
\mathbf{t}\left(s_{i}\right) & =\frac{\mathbf{c}\left(s_{i+1}\right)-\mathbf{c}\left(s_{i}\right)}{\left|\mathbf{c}\left(s_{i+1}\right)-\mathbf{c}\left(s_{i}\right)\right|}, \\
\mathbf{n}\left(s_{i}\right) & =\frac{\mathbf{b}_{r}\left(s_{i}\right)-\mathbf{c}\left(s_{i}\right)}{\left|\mathbf{b}_{r}\left(s_{i}\right)-\mathbf{c}\left(s_{i}\right)\right|}, \\
\mathbf{m}\left(s_{i}\right) & =\mathbf{t}\left(s_{i}\right) \times \mathbf{n}\left(s_{i}\right), \\
R\left(s_{i}\right) & =\left[\mathbf{t}\left(s_{i}\right) \mathbf{n}\left(s_{i}\right) \mathbf{m}\left(s_{i}\right)\right], \\
\dot{R}\left(s_{i}\right) & =\frac{1}{\Delta s_{i}}\left(R\left(s_{i+1}\right)-R\left(s_{i}\right)\right) .
\end{aligned}
$$

The generalised curvature vector $\Omega_{B}\left(s_{i}\right)$ is determined from $S\left(\Omega_{B}\left(s_{i}\right)\right)=\left(R^{T} \dot{R}\right)\left(s_{i}\right)$. Finally, the road width on either side of the centre line is approximated by

$$
\begin{aligned}
& n_{l}\left(s_{i}\right)=\mathbf{n}\left(s_{i}\right) \cdot\left(\mathbf{b}_{l}\left(s_{i}\right)-\mathbf{c}\left(s_{i}\right)\right) \\
& n_{r}\left(s_{i}\right)=\mathbf{n}\left(s_{i}\right) \cdot\left(\mathbf{b}_{r}\left(s_{i}\right)-\mathbf{c}\left(s_{i}\right)\right) .
\end{aligned}
$$

The unit vector $\mathbf{n}\left(s_{i}\right)$ comes from (26). The danger with this approach is explicit in (6) and (7), which show that the determination of curvature-type variables require that position variables be differentiated at least twice; this process is not viable when noise is present in the underlying measurements.

\subsection{Noise Influences}

Noisy road-boundary measurements can have a negative impact on the solution of related vehicular simulation and optimal control problems. As is evident from equations such as (6) and (7), boundary noise will re-appear (amplified by multiple differentiation operations) in road models such as those described in Section 2.4.

When numerical methods are used to solve the equations of motion, particularly if adaptive step size algorithms are used, modelling noise can take on the guise of 'fast dynamics' resulting in foreshortened integration step lengths [25], [26] and [27]. Reduced integration step lengths will increase the computation times and undermine accuracy, and these effects must be managed. In optimal control, mesh refinement schemes are used to improve both speed and accuracy [28]. In pseudospectral methods for optimal control variable order orthogonal

\footnotetext{
${ }^{3}$ Interpolation can be used to enforce this condition if the number of measurement points for the two boundaries is different.
} 
polynomials, which are used in Gaussian quadrature schemes, are used for the same purpose [29]. Again, noise in the road model will drive erroneously the meshing and polynomial order adaptation algorithms. Since noise disrupts both the numerical integration and the optimal control solution processes it should be removed where possible.

Another issue relates to the introduction of drift phenomena in road modelling. As has been demonstrated in [2], classical filtering techniques can be used to remove noise, but these processes tend to introduce drift that changes the road geometry and prevents circuit closure. In this case the circuit's beginning and end are no longer at the same point in space.

As we will now show, an appropriately formulated optimal control problem can be used to reject measurement noise from the road model, while simultaneously guaranteeing track closure. In essence, we propose the use of an optimal filtering process with boundary constraints.

\subsection{Problem Formulation}

The track-smoothing optimal control problem is embedded in the following general framework:

$$
\begin{gathered}
\min _{\left\{v(\cdot), \chi\left(s_{0}\right)\right\}} \int_{s_{0}}^{s_{f}} l(s, \chi(s), v(s)) d s, \\
\text { such that }\left\{\begin{array}{l}
\dot{\chi}=f(s, \chi(s), v(s)) \\
0 \geq h(s, \chi(s), v(s)) \\
0=g_{b}\left(\chi\left(s_{0}\right), \chi\left(s_{f}\right)\right) .
\end{array}\right.
\end{gathered}
$$

The state and the control vectors are denoted $\chi(s)$ and $v(s)$ respectively ${ }^{4}$ The system dynamics are described by the vector-valued equation $\dot{\chi}=f(s, \chi, v)$. The vector-valued function $h(s, \chi, v)$ and the scalar function $l(s, \chi, v)$ define the path inequality constraints and the integral cost, respectively, with the boundary equality constraints described by the vector-valued function $g_{b}\left(\chi\left(s_{0}\right), \chi\left(s_{f}\right)\right)$.

\subsubsection{Dynamics}

A dynamic model based on the Euler angles $\theta, \mu$ and $\phi$ is used to represent the ribbon. The coordinates of the origin of the moving ribbon trihedron are given by $x, y$ and $z$. The right- and left-hand track widths are given by $n_{l}(s)$ and $n_{r}(s)$. The state equations are based on (24), with the state vector given by

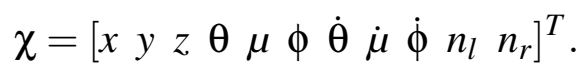

The control vector is

$$
v=\left[\begin{array}{lllll}
\ddot{\theta} & \ddot{\mu} & \ddot{\phi} & \dot{n}_{l} & \dot{n}_{r}
\end{array}\right]^{T} \text {. }
$$

The ribbon is generated by the control variables and the system dynamics

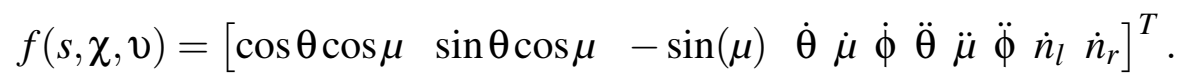

When the model is in this form, state and input constraints can be used to limit the velocities and accelerations of the Euler angles, and the velocity and magnitude variations in the track width.

\footnotetext{
${ }^{4}$ Contrary to accepted convention we use $\chi$ to represent the state vector and $v$ to denote the control vector. This notation is used to avoid confusion with the first translational component $x$ of a position vector, and with the longitudinal velocity $u$ of a vehicle as is used in Part 2 [30].
} 


\subsubsection{Cost}

The aim of the optimal control problem is to minimise the Euclidean distances between the ribbon model given in (29), (30) and (31), and the measurement-related data sets $\overline{\mathcal{B}}_{l}, \overline{\mathcal{B}}_{r}$ and $\overline{\mathcal{C}}$. The coordinates of the ribbon spine are given by the arc-length-dependent states $x, y$ and $z$, while the coordinates of the boundaries are the system outputs $\mathbf{b}_{l}$ and $\mathbf{b}_{r}$ as described by

$$
\begin{aligned}
& \mathbf{x}=\left[\begin{array}{lll}
x & y & z
\end{array}\right]^{T}, \\
& \mathbf{b}_{l}=\mathbf{x}+\mathbf{n} n_{l}, \\
& \mathbf{b}_{r}=\mathbf{x}+\mathbf{n} n_{r},
\end{aligned}
$$

with $\mathbf{n}$ given by the second column of (19).

The integral cost function $l(s, \chi, v)$ in $(28)$ is made up of three terms

$$
l(s, \chi, \mathrm{v})=e(s, \chi, \mathrm{v})+r_{c}(s, \mathrm{v})+r_{w}(s, \mathrm{v})
$$

The first term in $l(s, \chi, v)$ describes the tracking error, which also contains three terms as follows

$$
e(s, \chi, v)=w_{c}\|\mathbf{x}-\overline{\mathbf{c}}\|^{2}+w_{l}\left\|\mathbf{b}_{l}-\overline{\mathbf{b}}_{l}\right\|^{2}+w_{r}\left\|\mathbf{b}_{r}-\overline{\mathbf{b}}_{r}\right\|^{2}
$$

in which $w_{c}, w_{l}$ and $w_{r}$ are weighting functions.

The first term in (34) describes the error between the spine curve $\mathbf{x}$ and the road centre line $\overline{\mathbf{c}}$, which is a curve that interpolates the spline data. The second and third terms relate to the errors between the ribbon edges and curves and $\overline{\mathbf{b}}_{l}$ and $\overline{\mathbf{b}}_{r}$, which interpolate the track boundary data.

The second term in (33) is used to attenuate the high-frequency content in the ribbon curvatures. This is achieved through the curvature rate term $r_{c}(s, v)$, which is defined as the weighted sum of the squares of the second derivatives of the Euler angles

$$
r_{c}(s, v)=w_{\theta} \ddot{\theta}^{2}(s)+w_{\mu} \ddot{\mu}^{2}(s)+w_{\phi} \ddot{\phi}^{2}(s)
$$

in which $w_{\theta}, w_{\mu}$ and $w_{\phi}$ are again weighting terms.

The third term in (33) is used to remove high-frequency content (noise) from the track boundaries. The width rate is defined as the weighted sum of the squared derivatives of the left- and right-hand side offsets

$$
r_{w}(s, v)=w_{n_{l}} \dot{n}_{l}^{2}(s)+w_{n_{r}} \dot{n}_{r}^{2}(s)
$$

$w_{n_{l}}$ and $w_{n_{r}}$ are weighting terms.

The eight weighting factors are used to quantify the relative importance of the various influences in the optimal control problem. In broad terms, more accurate tracking is obtained with larger weighting factors in (34), whereas larger weights in (35) and (36) lead to smoother results. These weights are design parameters that have to be tuned in every application. In some optimal control problems a boundary cost term is included in (28), but this is not necessary in our case since all the boundary conditions are dealt with by the constraint terms. 


\subsubsection{Constraints}

As mentioned in Section 2.4 singular configurations must be avoided. In order to exclude Euler-angle-related singularities from the optimal control problem, the following box constraint is placed on the pitch angle: $-\pi / 2<$ $\mu<\pi / 2$, which limits the absolute value of the modelled road slope.

Boundary constraints on the initial and final positions and orientations of the ribbon trihedron will be applied. Such constraints are of the form $\chi\left(s_{0}\right)=x_{0}$ and $\chi\left(s_{f}\right)=x_{f}$, where the initial and final references $x_{0}$ and $x_{f}$ are found $^{5}$ using the procedure in Section 3.1. In some cases the initial and final values of the states may be left unconstrained, relying on the optimisation process to establish optimal values for these variables.

When modelling closed road circuits, such as race tracks, periodicity constraints are required. In this case one must enforce $\chi\left(s_{f}\right)=\chi\left(s_{0}\right)$, which ensures continuity of the state across the start-finish line.

Note that the periodicities in the angles may have to be accommodated, which allow the boundary angles to differ by integer multiples of $2 \pi$. For 'real' closed circuits the pitch and roll angles are identical at the boundaries. However, if an integer number of complete laps ( $k$ say) is needed in the problem set up, a constraint of the form $\theta\left(s_{f}\right)-\theta\left(s_{0}\right)-2 k \pi=0$ may be required.

\section{Results}

The optimal control problem formulated in Section 3.3 is used to parameterise two ribbon models. The first is illustrative and demonstrates the optimal control solution method for a three-dimensional ribbon with a known analytical parameterisation. In the second example we find a parameterisation of the "Circuit de Catalunya" in Barcelona, Spain. The boundary points for the race track are in the form of GPS data. The generalised curvature vector and the distances of the boundaries from the ribbon spine are derived using an optimal control calculation. The continuous-time optimal control problems are solved using a direct method for optimal control, in which the problem differential equations are transcribed into difference equations using an approximation scheme. In each case the solution of the resulting discrete-time optimal control problem is then found using Non-Linear Programming techniques. The toolbox GPOPS-II [29,31,32], implemented in MATLAB, was used for the solutions presented in this work. GPOPS-II uses pseudospectral discretisation and a nonlinear programme solver to obtain solutions to optimal control problems. It employs an $h p$-adaptive Gaussian quadrature integration scheme with the collocation performed at the Legendre-Gauss-Radau quadrature points. A sparse finite-differencing process is employed to estimate all the first- and second-order derivatives required by the NLP solver.

\subsection{Lemniscate Ribbon}

The illustrative ribbon considered here is based on the Lemniscate of Bernoulli [33], which we have augmented with an elevation dimension in order to generate a spatial spine curve. A camber angle is introduced that describes the direction in which the ribbon's width evolves.

The original lemniscate is a plane curve defined as the locus of points for which the product of the distances from two fixed foci at $x= \pm c$ is constant:

$$
\left((x-c)^{2}+y^{2}\right)\left((x+c)^{2}+y^{2}\right)=c^{4} .
$$

In the case that $c=1 / \sqrt{2}$, the lemniscate is described parametrically as

$$
\begin{aligned}
& x(p)=\frac{\cos (p)}{1+\sin (p)^{2}}, \\
& y(p)=\frac{\sin (p) \cos (p)}{1+\sin (p)^{2}},
\end{aligned}
$$

\footnotetext{
${ }^{5}$ The forward Euler scheme for finite differences can be used for $x_{0}$, whereas $x_{f}$ has to be defined using a backward finite-differentiation rule.
} 
for $p \in[0,2 \pi]$. It is well known that the $p$-parameter and the lemniscate arc length are related by an incomplete elliptic integral of the first kind. Such integrals must be evaluated numerically and so the differential-geometric properties of lemniscate-related curves are also best computed numerically.

If the following $z$-coordinate is introduced

$$
z(p)=\frac{1}{10} \sin (p)
$$

one obtains a three-dimensional space curve in the form of a figure of eight. In our first example this space curve is used as the spine of a ribbon that is then augmented with a symmetric width of the form

$$
n_{r}(p)=-n_{l}(p)=\frac{1}{20}\left(1+\frac{1}{2} \cos (2 p)\right)
$$

The direction of the camber vector $\mathbf{n}(p)$, which is orthogonal to the curve tangent $\mathbf{t}(p)$, is deemed to lie at an angle of

$$
\mathrm{v}(p)=\cos (p)
$$

relative to the inertial $x y$-plane. This camber is measured around $\mathbf{t}$ with a right-hand rule. Figure 2 shows the ribbon surface as a grey-shaded membrane. The ribbon spine $\mathcal{C}$ and its boundaries $\mathcal{B}_{l}$ and $\mathcal{B}_{r}$ are given by

$$
\begin{aligned}
\mathbf{x} & =\left[\begin{array}{lll}
x & y & z
\end{array}\right]^{T}, \\
\mathbf{b}_{l} & =\mathbf{x}+n_{l} \mathbf{n}, \\
\mathbf{b}_{r} & =\mathbf{x}+n_{r} \mathbf{n} .
\end{aligned}
$$

When the optimal estimation problem is solved, the simulated data sets are of the form

$$
\begin{aligned}
\overline{\mathbf{b}}_{l}\left(s_{i}\right) & =\mathbf{b}_{l}\left(s_{i}\right)+\Delta_{l}, \\
\overline{\mathbf{b}}_{r}\left(s_{i}\right) & =\mathbf{b}_{r}\left(s_{i}\right)+\Delta_{r},
\end{aligned}
$$

in which $\mathbf{b}_{l}\left(s_{i}\right)$ and $\mathbf{b}_{r}\left(s_{i}\right)$ are sampled values of $\mathbf{b}_{l}$ and $\mathbf{b}_{r}$, respectively, at each sample point $s_{i}$. The sequences $\Delta_{l}$ and $\Delta_{r}$ are realisations of additive measurement noise processes that will be described below.

In order to test the smoothing capabilities of the optimal smoothing process discussed in Section 3 , we will begin by sampling the noise-corrupted boundary curves described in (43). The six components of the noise processes $\Delta_{l}$ and $\Delta_{r}$ are assumed to be uniformly distributed. The data associated with the optimal control problem is therefore a finite set of $p$-equispaced boundary samples computed from (43), in which the components of $\Delta_{l}$ and $\Delta_{r}$ are independent realisations of $U(-.01, .01) ; U(\cdot, \cdot)$ is a uniform distribution with prescribed limits. The maximum noise-related displacement of each boundary point is thus $\sqrt{3} / 100$, with the minimum track width given by $5 / 100$.

The three sets of weighting factors given in Table 1 will be considered in order to demonstrate their influence on the estimated curvature parameters.

The solution to the optimal estimation problem for the weight combination (a) is shown in Figure 2 . It is evident that the spine and boundary curves are 'smooth' in comparison with the raw reference data on which the optimal parameter estimates are based. The three differential-geometric parameters (generalised curvatures) for 


\begin{tabular}{c|cccc|c} 
& $w_{c}, w_{l}, w_{r}$ & $w_{\theta}, w_{\phi}$ & $w_{\mu}$ & $w_{n_{l}}, w_{n_{r}}$ & Plot colour \\
\hline \hline (a) & 100 & 0.001 & 0.05 & 1 & Black \\
(b) & 100 & 0.1 & 5 & 1 & Green \\
(c) & 100 & 0.001 & 0.05 & 50 & Blue
\end{tabular}

Table 1. Optimisation weights.

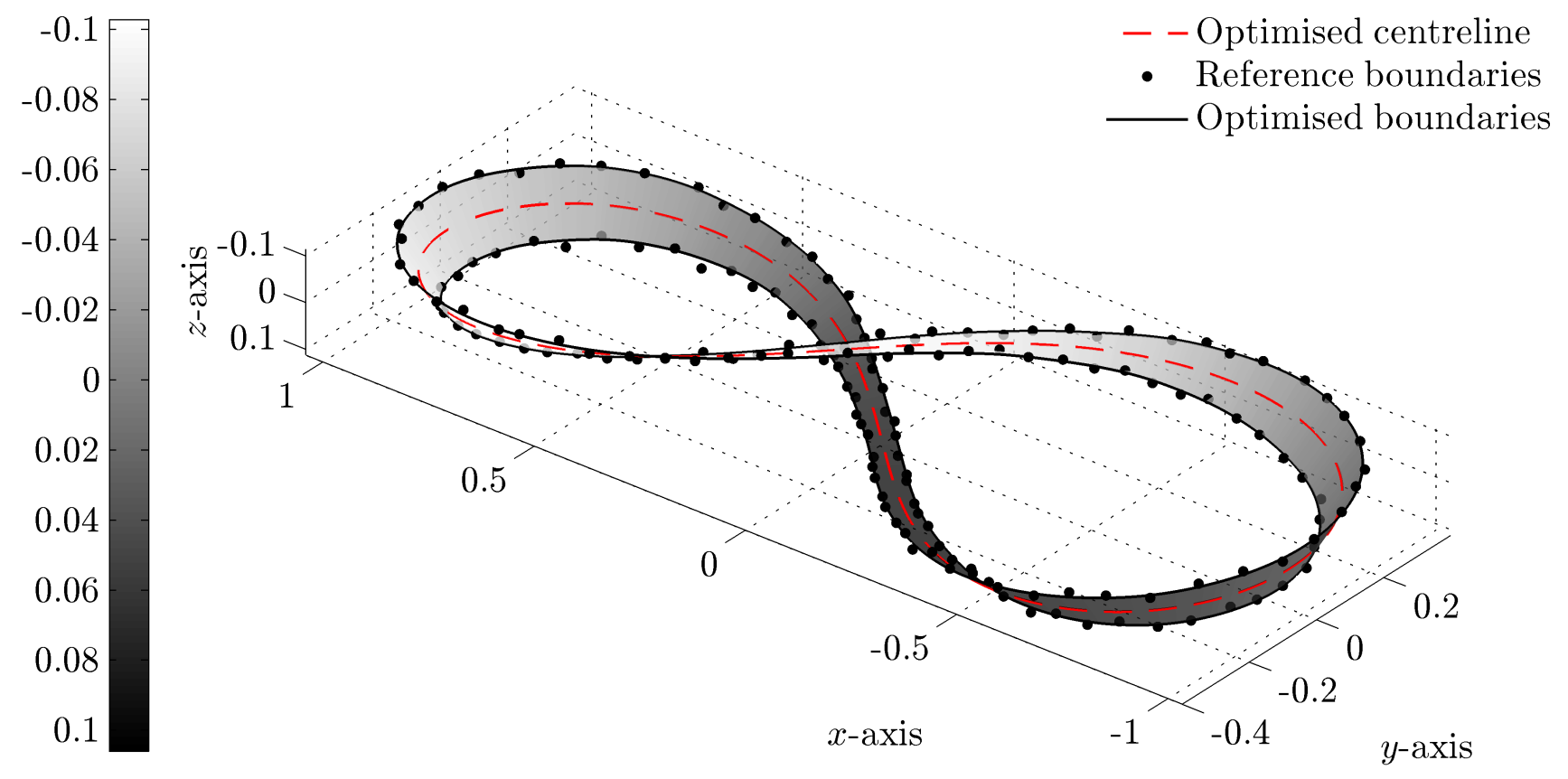

Fig. 2. Three-dimensional ribbon for the extended Lemniscate of Bernoulli. The solid and dashed lines are the optimised boundaries and centreline, respectively. The dots represent the noisy simulated boundary-point measurements on which the solution is based. The ribbon grey scale represents the $z$-coordinate of the optimised centreline and is calibrated according to the colourbar given.

the optimised ribbon spine are shown in Figure 3 using the colouring convention from Table 1. In each case the theoretical values and the optimal estimates are displayed, with good agreement between the two demonstrated for the weight selection (a) in Table 1.

The track width and its optimal estimates are also shown in Figure 3. The oscillations in the estimates around the true values are a consequence of the additive noise in the simulated boundary points; see (43). Figure 4 shows sums of the left- and the right-hand boundary estimation errors. The sum of the boundary tracking errors for the weight combination (a) is always less than 0.02 and on average lower than 0.01 , which is the limit set for the uniformly distributed random noise added to the reference boundaries. These properties of the boundary error suggest that good tracking is achieved.

In order to reduce the oscillations in the width parameters one could increase the $w_{n_{1}}$ and $w_{n_{2}}$ weighting coefficient (as defined in Section 3.3.2), but this would come at the cost of increased errors in the estimates, as is evident from the inspection of the blue curves in Figures 3 and 4, generated for the weight selection (c) in Table 1. If the smoothness of the generalised curvatures has priority over the tracking accuracy the values of 

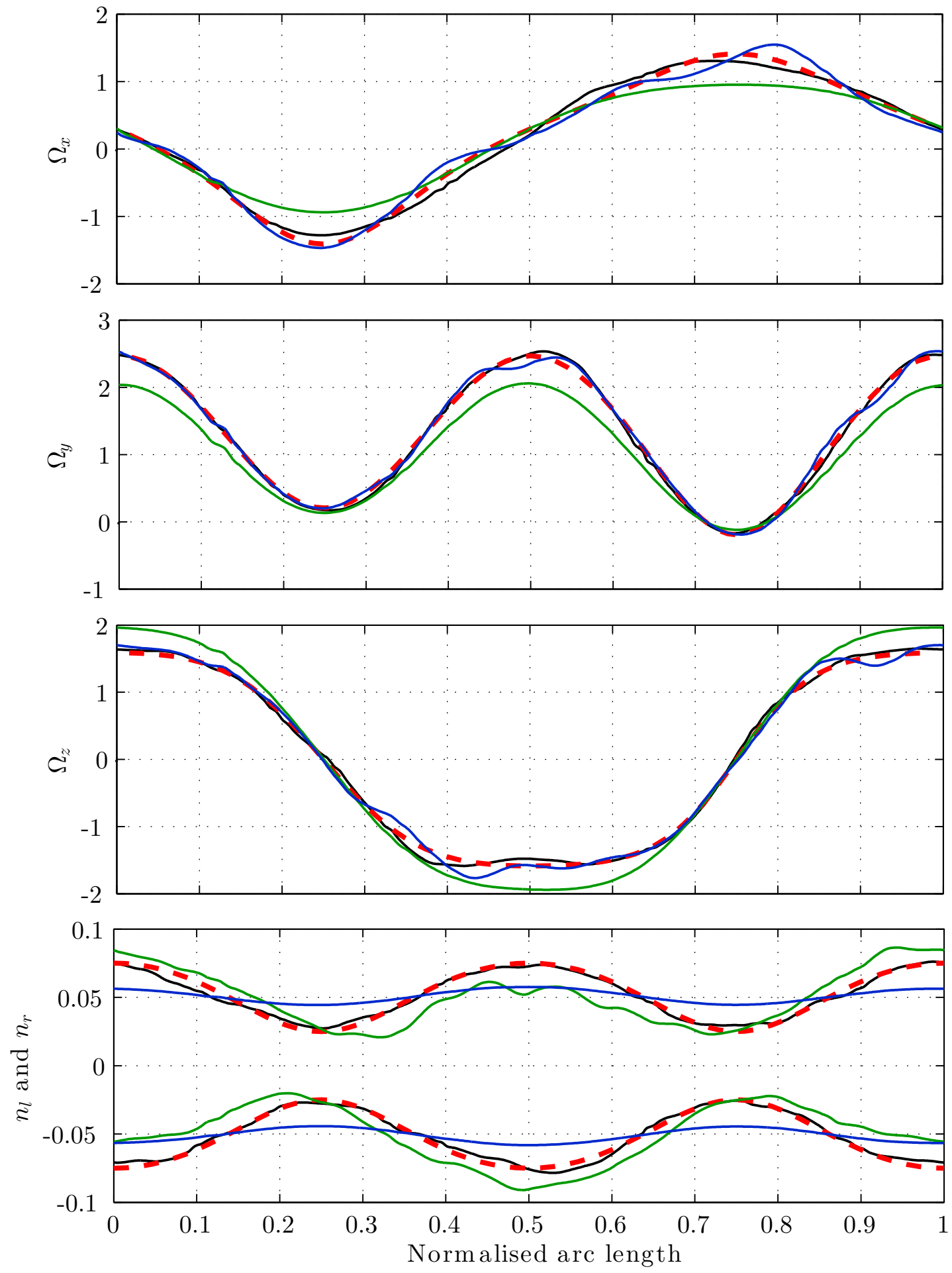

Fig. 3. Parameters for the extended lemniscate ribbon. The theoretically correct values are the red dashed curves, while the optimal estimates are the solid curves. The colour convention is the same as that given in Table 1. The top plot shows the relative torsion $\Omega_{x}$, the second the normal curvature $\Omega_{y}$, and the third the geodesic curvature $\Omega_{z}$. The distances between the ribbon boundaries and its spine are shown in the bottom diagram. The theoretical boundaries come from (40).

$w_{\theta}, w_{\phi}$ and $w_{\mu}$ may be increased. Figure 3 shows that the short-wavelength oscillations almost disappear from the identified curvatures for the weight combination (b) in Table 1 (green curves). As a consequence all the differential parameters and the track boundary estimates lose fidelity against the true theoretical values. It is clearly desirable to have some frequency separation between measurement noise and the characteristic frequencies of the track itself. It may be necessary to enforce this track-noise separation by collecting finer measurements (in terms of both 


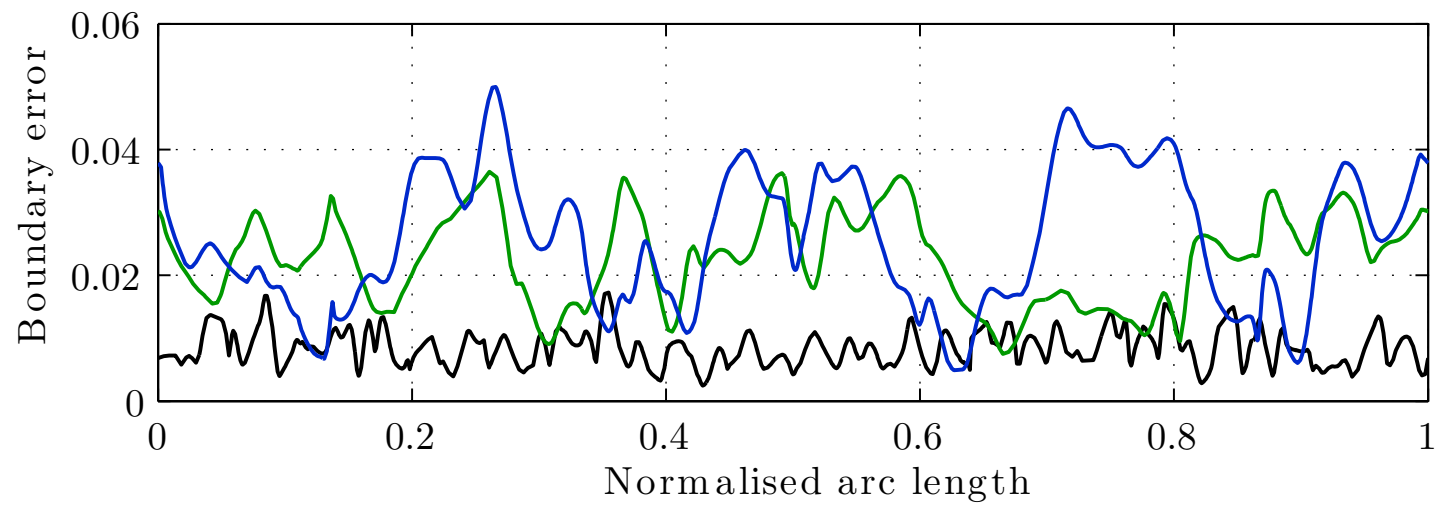

Fig. 4. Sums of the left- and right-hand boundary errors for different weight selections. The colour convention is the same as that used in Table 1 .

distance between measurement points and the measurement-noise filtering), especially where the track contains important higher-frequency features.

\subsection{Circuit de Catalunya}

The second application of the optimal ribbon parameterisation aims to model the 'Circuit de Catalunya', which is the current Spanish Formula 1 Grand Prix race track. The track boundary data were collected manually from satellite pictures combined with GPS coordinate data available from earth.google.com. The measured data points are not evenly spaced along the track edges. The elevation of each boundary point was found using altitude data from www.gpsvisualizer.com; the altitude variation around the track was found to be approximately $30 \mathrm{~m}$. This data, which is a combination of GPS and altitude information, was transformed into local SAE Cartesian coordinates using the MATLAB ${ }^{\mathrm{TM}}$ Geodetic Toolbox and the WGS84 geodetic system ${ }^{6}$. The inertial reference frame has its $x$ - and $y$-axes tangent to the earth's surface at a point inside the track perimeter, and with its $x$-axis parallel to the equator. The lowest point on the track was deemed to have an altitude of $0 \mathrm{~m}$. This process produced two three-dimensional data sets describing the tracks left- and right-hand boundaries. The circuit length is $4655 \mathrm{~m}$ and approximately three hundred data points were used for each track boundary.

For this problem the weights in the optimal control cost function (see Section 3.3.2) were chosen as: $w_{c}=$ $w_{l}=w_{r}=10^{-3}, w_{\theta}=3 \cdot 10^{3}, w_{\mu}=w_{\phi}=10^{9}$, and $w_{n_{l}}=w_{n_{r}}=10^{-2}$. These weights were chosen to best reflect to problem requirements and the units used. The weight selection is a design choice and will almost always require some form of trial-and-error tuning process. The optimal ribbon found via the solution of the optimal control problem is displayed in Figure 5. The corners where the maximum boundary tracking errors occur are shown in Figure 6. This figure illustrates clearly the effects of the smoothing process: the optimised boundary lines follow closely the measured boundary points, but without the introduction of sharp kinks, or rapid changes of curvature.

The next three figures provide a comparison between the differential-geometric parameters obtained through optimal control and the finite-difference approximation described in Section 3.1. It is clear that the optimally smoothed track parameters are more suitable for subsequent vehicular simulation and/or optimal-control studies. The relative torsion $\Omega_{x}$, the normal curvature $\Omega_{y}$ and the geodesic curvature $\Omega_{z}$ are shown in Figure 7 .

The track width of the optimised ribbon is represented in Figure 8. It appears that the track has an approximate width of twelve metres, with the exception of turn 10, where the width increases due to the corner's particular profile. Although the track width is almost constant through the corners 14 and 15, some oscillations appear in the estimates. This is due to the high geodesic curvature associated with these corners, where large curvature variations are smoothed by the optimal estimation process. In order to achieve this smoothing the ribbon axis is 'taking a racing line' through these corners causing oscillatory variations in the boundary-to-spine distances.

\footnotetext{
${ }^{6}$ The World Geodetic System is a standard for use in cartography and geodesy. It comprises a standard coordinate frame for the Earth, a datum or reference ellipsoid for altitude data and a gravitational equipotential surface (the geoid) that defines nominal sea level. The latest revision is WGS 84 that was last revised in 2004.
} 


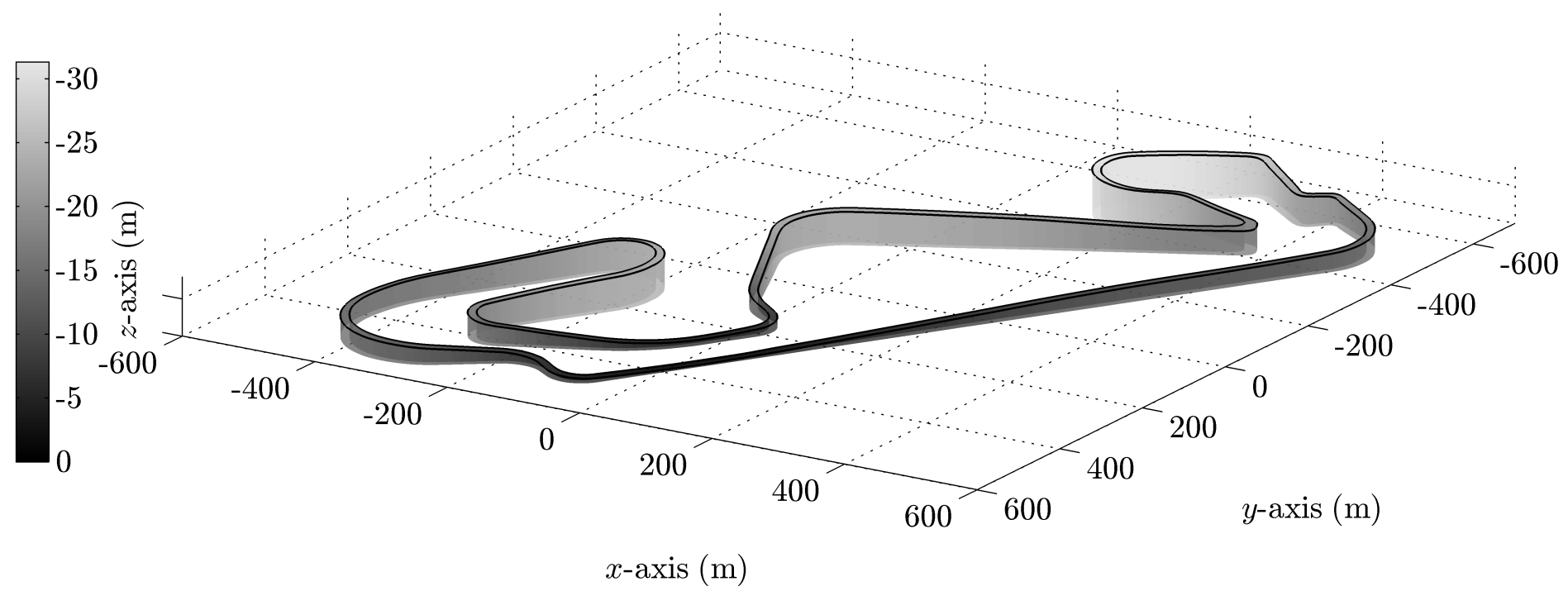

Fig. 5. Ribbon-based model of the Circuit de Catalunya. The solid lines are the optimised track boundaries. The track spine's z-coordinate is shown using a grey scale.
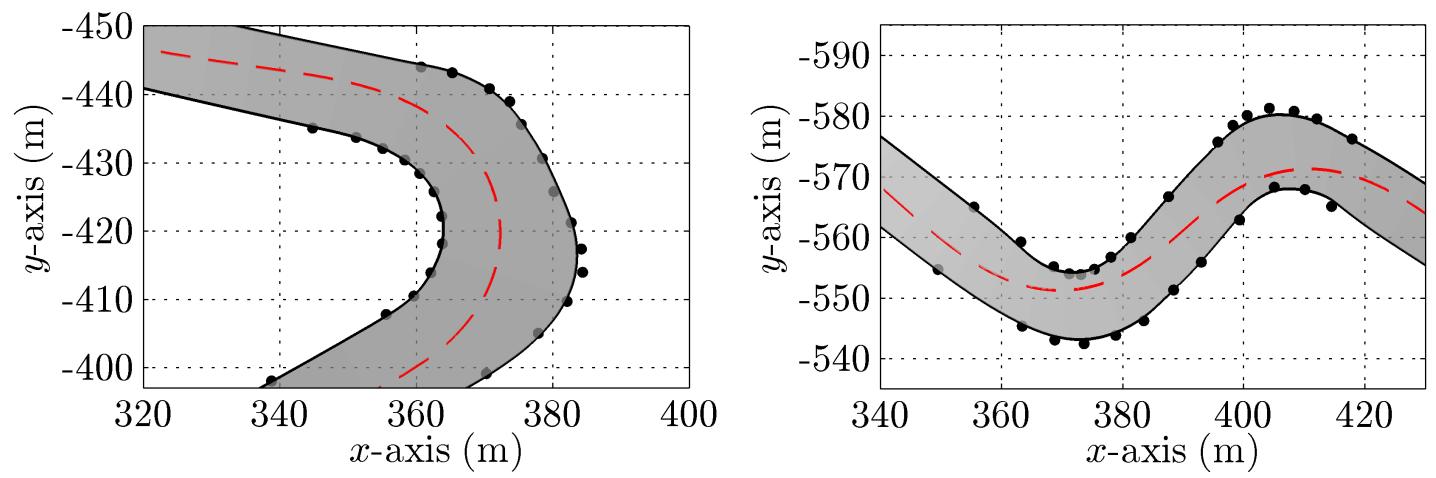

Fig. 6. Magnified views of corner 10 (left-hand side), and corners 14 and 15 (right-hand side). The measured boundary points are shown as black dots, with the dashed red curve the spine curve estimate.

Distance errors between the optimised track boundaries and the noisy measurement points are given in Figure 9. For the most part the estimated errors are less than $0.2 \mathrm{~m}$, which is comparable to the position accuracy expected of the measurement process used to generate the original data. The manual selection of the track boundaries was performed using satellite imagery at a resolution of approximately 2 pixels per metre; the expected measurement accuracy is not realistically better than $0.5 \mathrm{~m}$. Not unexpectedly, peaks in the boundary tracking errors correspond to corners 10,14 and 15. This is again a consequence of the high geodesic curvature through these corners. The magnified plot of the optimised boundaries given in Figure 6, shows that, locally, the inner boundary estimate still tracks the measured data smoothly and accurately. However, this comes at the price of poorer outer boundary estimates. In racing simulation and optimal control studies, small boundary modelling errors for the outer boundary are usually unimportant, because a racing line "cuts" the corners at the apex defined by the inner boundary in order to minimise the curvature of the vehicle's trajectory. 

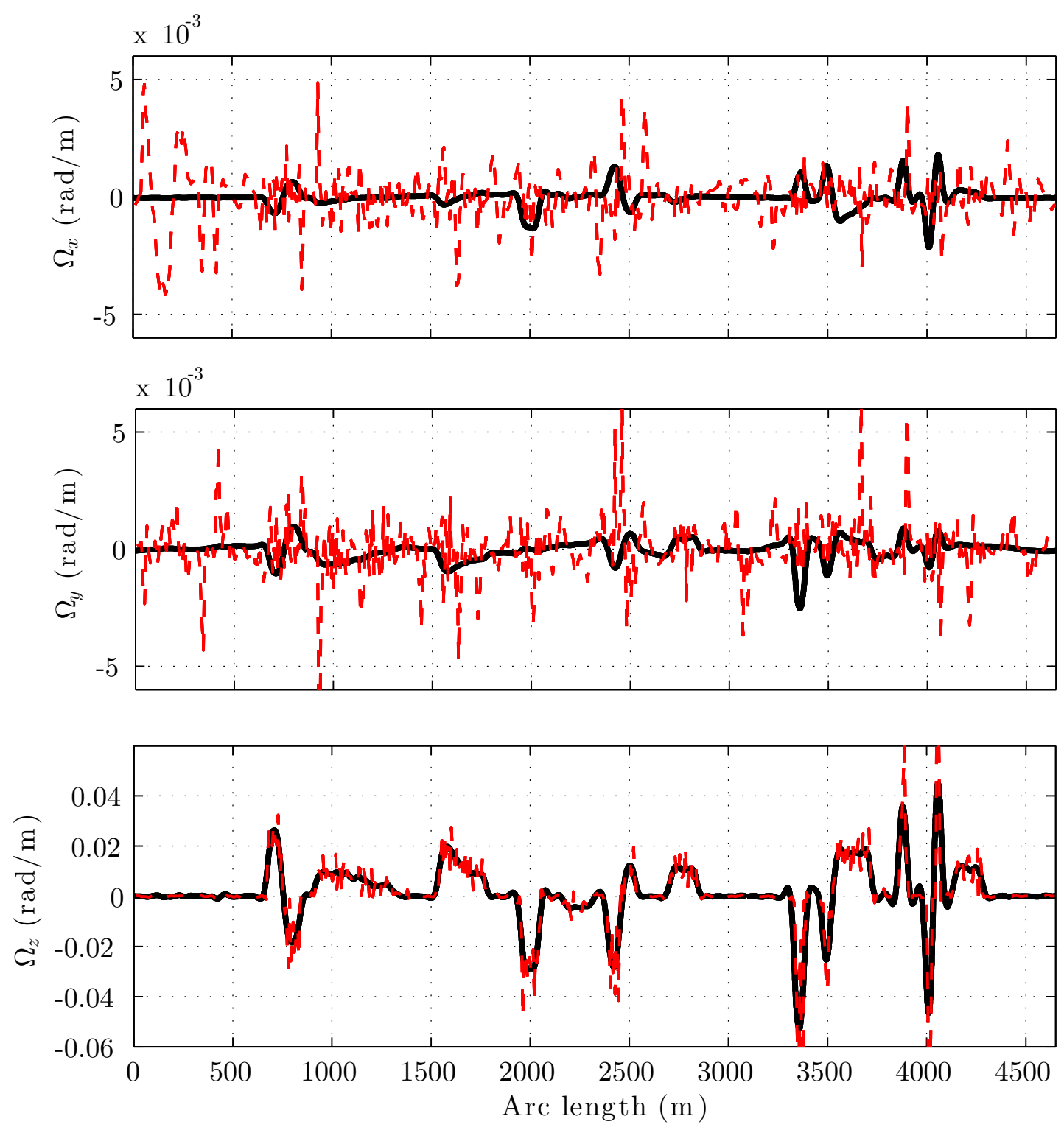

Fig. 7. Estimated curvature of the Circuit de Catalunya. The plots show, respectively, the relative torsion $\Omega_{x}$, the normal curvature $\Omega_{y}$ and the geodesic curvature $\Omega_{z}$ (in radians per meter). The optimal model-based estimates are shown as the solid black curves, while the finite-difference approximations are the red dashed curves.

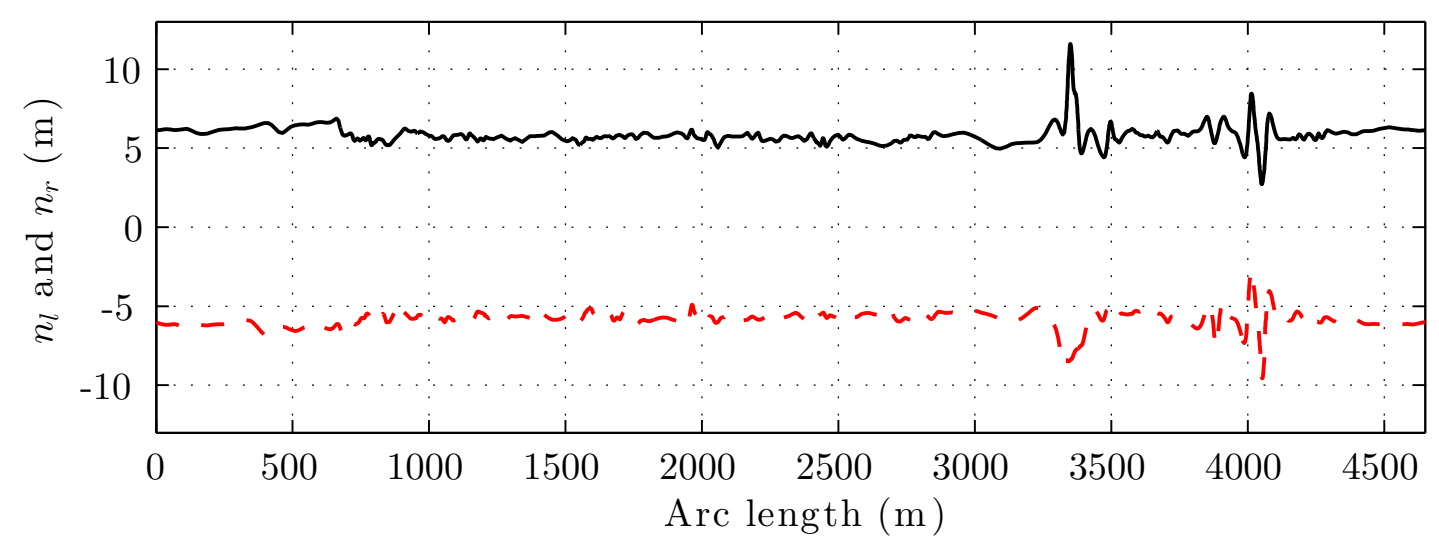

Fig. 8. Distances (in metres) to the right-hand (solid black) and left-hand (dashed red) boundaries of the track as measured from the track's spine. 


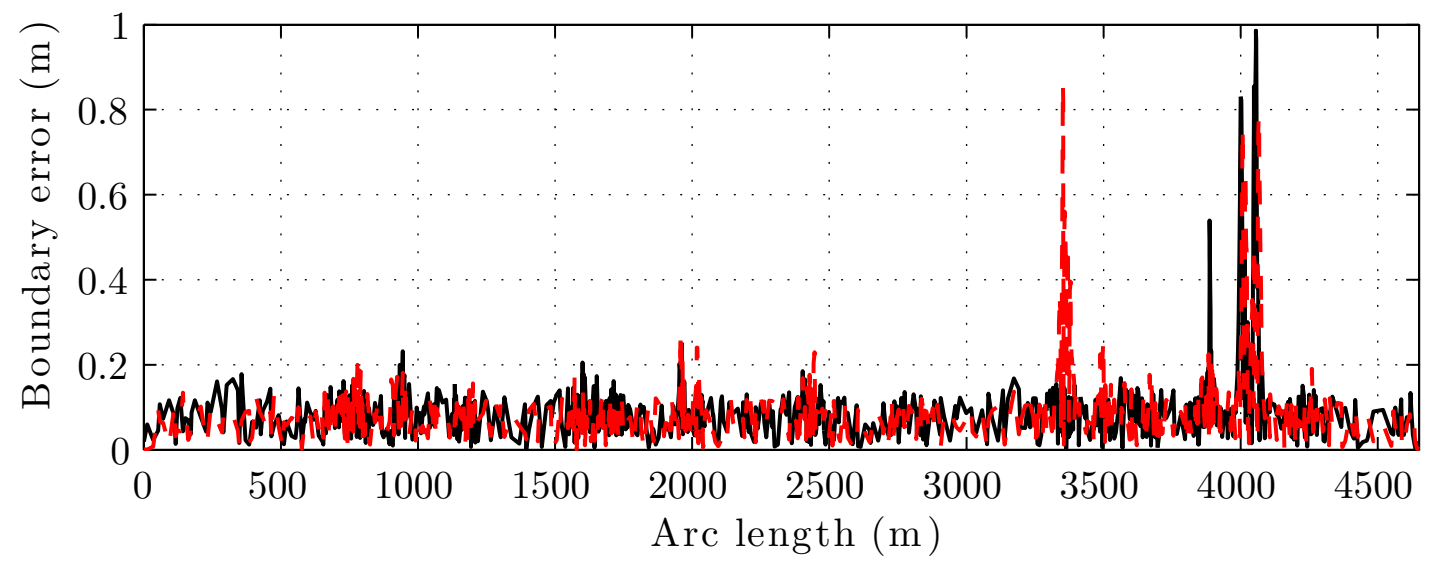

Fig. 9. Error estimates for the track boundaries (as calculated in the ribbon tangent plane in metres). The solid black curve corresponds to the right-hand-side boundary, while the dashed red curve corresponds to the left-hand boundary.

\section{Conclusions}

The aim of this work was to develop a method of estimating the geometric properties of roads and race tracks from discrete noisy data sets. Models of this type are useful in a wide variety of vehicular simulation and optimal control problems such a minimum-lap-time studies typically associated with closed-circuit racing. The method proposed is an optimal estimation process in which a generic geometric model of the roadway is assumed, with the unknown track curvature and boundary parameters deduced from discrete noisy data. Unlike standard white-box identification problems in which fixed parameters are sought, the unknown parameters in this case are curves. In the first part of the paper we showed how known ideas from differential geometry can be used to develop a generic track/road model, which is described in terms of three curvature functions and two track-width functions. We then show how the estimation of these (five) functions can be transformed into a numerical optimal control problem that can be solved by known means. The procedure is demonstrated on the illustrative problem of finding the parameters of a ribbon based on the Lemniscate of Bernoulli. It is also shown how the optimal estimation problem can be 'tuned' to reject measurement noise. The paper concludes with the more substantial problem of optimising the ribbon parameters of the Circuit de Catalunya. In this case the noisy raw data is derived from publicly available resources. We hope that we have convinced the reader that optimal-estimation-based processes of the type shown here are superior to filtering the data using ad-hoc techniques.

\section{Acknowledgments}

This work was supported by the UK Engineering and Physical Sciences Research Council.

\section{References}

[1] Hendrikx, J. M., Meijlink, T., and Kriens, R. F. C., 1996. "Application of optimal control theory to inverse simulation of car handling”. Vehicle System Dynamics, 26, pp. 449-461.

[2] Casanova, D., 2000. On Minimum Time Vehicle Manoeuvring: The Theoretical Optimal Lap. Cranfield University School of Engineering. PhD Thesis.

[3] Kelly, D. P., 2008. Lap Time Simulation with Transient Vehicle and Tyre Dynamics. Cranfield University School of Engineering. PhD Thesis.

[4] Cossalter, V., Lio, M. D., Lot, R., and Fabbri, L., 1999. "A general method for the evaluation of vehicle manoeuvrability with special emphasis on motorcycles". Vehicle System Dynamics, 31, pp. 113-135.

[5] Perantoni, G., and Limebeer, D. J., 2014. "Optimal control for a formula one car with variable parameters". Vehicle System Dynamics, 52(5), pp. 653-678. 
[6] Timings, J. P., and Cole, D. J., 2013. "Minimum maneuver time calculation using convex optimization". Journal of Dynamic Systems, Measurement, and Control, 135, pp. 031015-031015-9.

[7] Koenderink, J. J., 1990. Solid Shape. Artificial Intelligence. MIT Press.

[8] White, J. H., and Bauer, W. R., 1986. "Calculation of the twist and the writhe for representative models of DNA". Journal of Molecular Biology, 189(2), pp. 329 - 341.

[9] Panyukov, S., and Rabin, Y., 2000. "Fluctuating filaments: statistical mechanics of helices". Physical review. E, 62(5 Pt B), pp. 7135-46.

[10] Kessler, D. A., and Rabin, Y., 2003. "Effect of curvature and twist on the conformations of a fluctuating ribbon". The Journal of Chemical Physics, 118(2), pp. 897-904.

[11] Rappaport, S. M., and Rabin, Y., 2007. "Differential geometry of polymer models: worm-like chains, ribbons and fourier knots". J. Phys. A: Math. Theor., 40, pp. 4455 - 4466.

[12] Behringer, R., van Holt, V., and Dickmanns, D., 1992. "Road and relative ego-state recognition". In Intelligent Vehicles '92 Symposium., Proceedings of the, pp. 385-390.

[13] Dickmanns, E., and Mysliwetz, B., 1992. "Recursive 3-d road and relative ego-state recognition". Pattern Analysis and Machine Intelligence, IEEE Transactions on, 14(2), Feb, pp. 199-213.

[14] Behringer, R., 1995. "Detection of discontinuities of road curvature change by glr". In Intelligent Vehicles '95 Symposium., Proceedings of the, pp. 78-83.

[15] Khosla, D., 2002. "Accurate estimation of forward path geometry using two-clothoid road model". In Intelligent Vehicle Symposium, 2002. IEEE, Vol. 1, pp. 154-159 vol.1.

[16] Loose, H., and Franke, U., 2010. "B-spline-based road model for 3d lane recognition". In Intelligent Transportation Systems (ITSC), 2010 13th International IEEE Conference on, pp. 91-98.

[17] Cong, S., Shen, S., and Hong, L., 2009. Road curvature estimation system, Dec. 1. US Patent 7,626,533.

[18] Shen, T., and Ibrahim, F., 2012. "Interacting multiple model road curvature estimation”. In Intelligent Transportation Systems (ITSC), 2012 15th International IEEE Conference on, pp. 710-715.

[19] Eidehall, A., and Gustafsson, F., 2006. "Obtaining reference road geometry parameters from recorded sensor data”. In Intelligent Vehicles Symposium, 2006 IEEE, pp. 256-260.

[20] Mena, J., 2003. "State of the art on automatic road extraction for GIS update: a novel classification". Pattern Recognition Letters, 24(16), pp. 3037 - 3058.

[21] Lin, X., Zhang, J., Liu, Z., Shen, J., and Duan, M., 2011. "Semi-automatic extraction of road networks by least squares interlaced template matching in urban areas". International Journal of Remote Sensing, 32(17), pp. 4943-4959.

[22] Willemsen, P., Kearney, J., and Wang, H., 2003. "Ribbon networks for modeling navigable paths of autonomous agents in virtual urban environments". In Virtual Reality, 2003. Proceedings. IEEE, pp. 79-86.

[23] Kreyszig, E., 1991. Differential Geometry. University of Toronto Press.

[24] Struik, D. J., 1988. Lectures on Classical Differential Geometry, second ed. Dover, New York.

[25] Gear, C. W., 1971. Numerical initial value problems in ordinary differential equations. Prentice-Hall series in automatic computation. Prentice-Hall, Englewood Cliffs, New Jersey.

[26] Brenan, K. E., Campbell, S. L., and Petzold, L. R., 1996. Numerical Solution of Initial-Value Problems in Differential-Algebraic Equations. SIAM's Classics in Applied Mathematics. SIAM, Philadelphia.

[27] Griffiths, D., and Higham, D., 2010. Numerical Methods for Ordinary Differential Equations: Initial Value Problems. Springer.

[28] Betts, J. T., 2001. Practical methods for Optimal Control and estimation Using Nonlinear Programming, second ed. SIAM, Philadelphia, PA.

[29] Darby, C. L., Hager, W. W., and Rao, A. V., 2011. "An hp-adaptive pseudospectral method for solving optimal control problems". Optimal Control Applications and Methods, 32(4), pp. 476-502.

[30] Limebeer, D. J. N., and Perantoni, G., 2013. "Optimal control of a formula one car on a three-dimensional track part 2: Optimal control”. under review.

[31] Patterson, M. A., and Rao, A. V., 2013. “GPOPS - II: A matlab software for solving multiple-phase optimal 
control problems using hp-adaptive Gaussian quadrature collocation methods and sparse nonlinear programming”. ACM Transactions on Mathematical Software, under review.

[32] Patterson, M. A., and Rao, A. V., 2011. "Exploiting sparsity in direct collocation pseudospectral methods for solving optimal control problems". Journal of Spacecraft and Rockets, 49(2), pp. 364-377.

[33] Lawrence, J. D., 1972. A Catalog of Special Plane Curves. Dover Publications. 